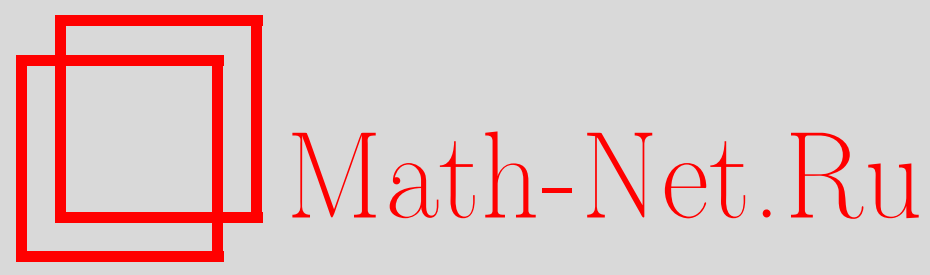

С. А. Дроздовский, Пример периферически счетного локально связного континуума, не являющегося линейно связным, Матем. заметки, 1999, том 65, выпуск 5, 659-666

DOI: https://doi.org/10.4213/mzm1097

Использование Общероссийского математического портала Math-Net.Ru подразумевает, что вы прочитали и согласны с пользовательским соглашением http://www.mathnet.ru/rus/agreement

Параметры загрузки:

IP : 54.224 .187 .69

26 апреля 2023 г., $17: 17: 22$

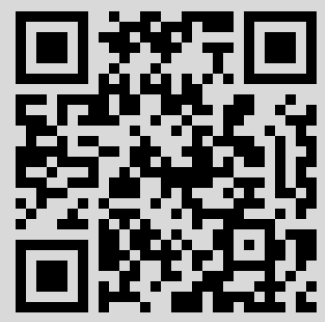




\section{ПРИМЕР ПЕРИФЕРИЧЕСКИ СЧЕТНОГО ЛОКАЛЬНО СВЯЗНОГО КОНТИНУУМА, НЕ ЯВЛЯЮШЕГОСЯ ЛИНЕЙНО СВЯЗНЫМ}

\section{С. А. Дроздовский}

Построен пример периферически счетного локально связного континуума, не являющегося линейно связным, имеющий значение для классификации локально связных континуумов. Результат интересен тем, что метризуемых локально связных континуумов, обладающих такими свойствами, не существует.

Библиография: 1 название.

Изложенный в статье результат касается классификации локально связных континуумов по свойствам типа связности, в частности, по периферическим свойствам.

ОПРЕДЕЛЕНИЕ. Топологическое пространство $X$ обладает периферическим свойством $\mathcal{P}$, если существует база открытых множеств пространства $X$,границы всех элементов которой обладают свойством $\mathcal{P}$.

В [1] дается подробная информация о соотношениях между классами локально связных континуумов. Там же ставится проблема: $\mathcal{R} \subseteq \mathcal{A}^{\prime}$ ? Здесь $\mathcal{R}$ - класс всех периферически счетных локально связных континуумов, $\mathcal{A}^{\prime}$ - класс всех линейно связных локально связных континуумов. Для метризуемых локально связных континуумов, которые достаточно хорошо изучены, постановка такого вопроса не имеет смысла.

Построенньй ниже пример решает данную проблему отрицательно.

Описание конструкции. Построим по индукции множество, являющееся объединением счетного семейства $\left\{\left[a_{i_{n}}, b_{i_{n}}\right): i_{n}=j_{0} j_{1} \ldots j_{n}, j_{0}=0,1 \leqslant j_{k}<\omega, 1 \leqslant k<\right.$ $\omega, n<\omega\}$ прямолинейных полуинтервалов, лежащих в плоскости $\mathbb{R}^{2}$. Сначала определим множество $L^{1}=\bigcup\left\{\left[a_{i_{n}}, b_{i_{n}}\right): n \leqslant 1\right\}$ (рис. 1 ). Длина отрезка $a_{0} b_{0}$ равна 1 . Множество $\left\{a_{0 j}: 1 \leqslant j<\omega\right\} \subset\left[a_{0}, b_{0}\right)$ является последовательностью, сходящейся к точке $b_{0}$. Для каждого $j$ расстояние $\rho\left(a_{0 j}, b_{0}\right)=1 / 2^{j}$, а расстояние $\rho\left(a_{0 j}, b_{0 j}\right)=1 / 4^{j}$. Все полуинтервалы $\left[a_{0 j}, b_{0 j}\right)$ перпендикулярны $a_{0} b_{0}$ и направлены в одну сторону. Пусть построено множество $L^{m}=\bigcup\left\{\left[a_{i_{n}}, b_{i_{n}}\right): n \leqslant m\right\}, m \geqslant 1$. Для каждого $i_{m}$ однозначно определяется отображение геометрического подобия $\theta_{i_{m}}$, отображающее $L^{1}$ в $\mathbb{R}^{2}$, такое, что $\theta_{i_{m}} a_{0}=a_{i_{m}}, \theta_{i_{m}} b_{0}=b_{i_{m}}$, и каждый полуинтервал $\left[a_{i_{m} j}, b_{i_{m} j}\right), a_{i_{m} j}=$ $\theta_{i_{m}} a_{0 j}, b_{i_{m} j}=\theta_{i_{m}} b_{0 j}$, сонаправлен с полуинтервалом $\left[a_{i_{m-1}}, b_{i_{m-1}}\right), i_{m}=i_{m-1} j_{m}$; $L^{m+1}$ по определению есть $\bigcup_{i_{m}} \theta_{i_{m}} L^{1} \cup L^{m}$. Осуществив последовательно построение 

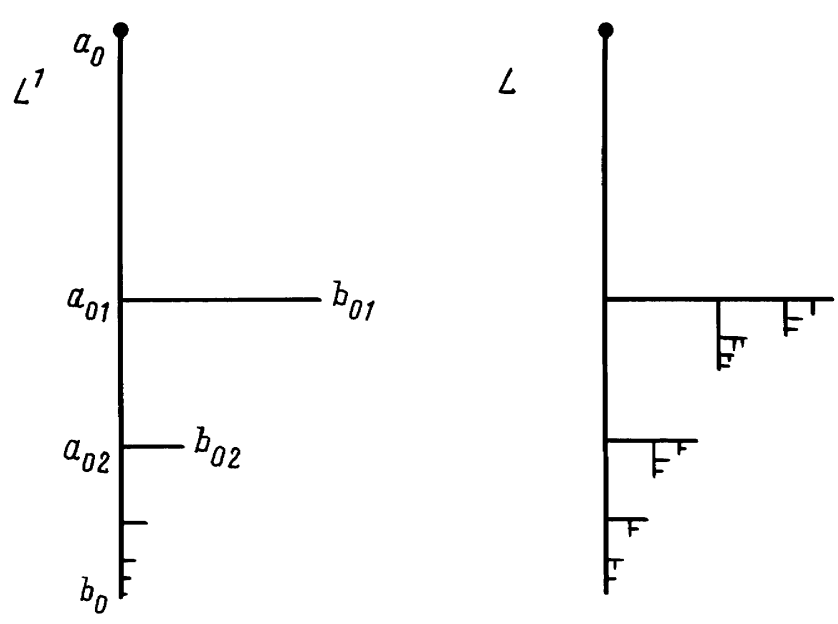

Рис. 1

всех множеств $L^{m}$ для $m<\omega$, мы получим линейно связное локально связное периферически конечное множество $L=\bigcup_{m} L^{m}$.

Замыкание множества $L$ в плоскости представляет собой компакт $M=L \cup Q$, где $Q=\bigcup_{i}\left\{q_{i}\right\}$ состоит из счетного множества "рациональных" точек $q_{i}=b_{i_{n}}, i=i_{n}$, и множества "иррациональных" точек $q_{i}, i=j_{0} j_{1} \ldots j_{k} \ldots$, мощности $c$. Каждая "рациональная" точка $q_{i_{n}}, i_{n}=j_{0} j_{1} \ldots j_{n}$, возникает при замькании в $\mathbb{R}^{2}$ ломаной $P_{i_{n}}=$ $a_{j_{0}} a_{j_{0} 1} a_{j_{0}} 2 \ldots a_{j_{0} j_{1}} \ldots a_{i_{n}} a_{i_{n} 1} a_{i_{n} 2} a_{i_{n}} \ldots$, , состоящей из счетного числа звеньев, которые все за исключением конечного подмножества лежат на одной прямой. Каждая "иррациональная" точка $q_{i}, i=j_{0} j_{1} j_{2} \ldots$, получается при замыкании ломаной счетного числа звеньев $P_{i}=a_{j_{0}} a_{j_{0} 1} a_{j_{0}} \ldots a_{j_{0} j_{1}} \ldots a_{j_{0} j_{1} j_{2}} \ldots$ В дальнейшем принципиальных различий между "рациональными" и "иррациональньпи" точками возникать не будет. $M$ является, очевидно, континуумом, обладающим перечисленными вьше свойствами множества $L$.

Пусть $\mathcal{A}=\left\{A_{i_{n}}^{j}: b_{i_{n}} \in Q, 0 \leqslant j<\omega\right\}$ - счетное покрытие множества $M$ замкнутыми квадратами $A_{i_{n}}^{j}$ с центрами в точках $b_{i_{n}}$ и со сторонами длины $4 \rho\left(b_{i_{n}}, a_{i_{n} j}\right)$, $a_{i_{n} 0}=a_{i_{n}}$, параллельными отрезкам, принадлежащим $M$ (рис. 2 ). В каждый квадрат $A_{i_{n}}^{j}$ впишем ортогональную сетку $B_{i_{n}}^{j}$, состоящую из включающего стороны квадрата конечного семейства отрезков, параллельных сторонам квадрата, не пересекающихся с множеством $Q$. Мы имеем множество $B=\bigcup\left\{B_{i_{n}}^{j}: A_{i_{n}}^{j} \in \mathcal{A}\right\}$. Ячейкой сетки мы будем назьвать какую-либо замкнутую ломаную, содержащуюся в $B$, а мелкостью $\delta(y)$ сетки в точке $y \in \mathbb{R}^{2}$ - точную нижнюю грань диаметров ячеек, содержащих $y$ в своей внутренней области, т.е. в той из двух связньх множеств плоскости, на которые разбивает ячейка плоскость согласно теореме Жордана, которая ограничена. Если точка $y$ не содержится во внутренней области никакой из ячеек, будем считать, что $\delta(y)=\infty$. Мелкость $\delta(E)$ сетки $B$ на каком-либо множестве $E \subset \mathbb{R}^{2}$ по определению равна $\sup _{y \in E} \delta(y)$. Мелкости сеток $B_{i_{n}}^{j}$, т.е. величины $\delta\left(A_{i_{n}}^{j}\right)$, мы выберем позднее. Расположение семейст- 
ва $\mathcal{A}$ на плоскости таково, что $[B]_{\mathbb{R}^{2}} \backslash B=Q$. Компакт $N=[L \cup B]_{\mathbb{R}^{2}}=L \cup B \cup Q$, очевидно, обладает всеми перечисленньми свойствами компакта $M$.
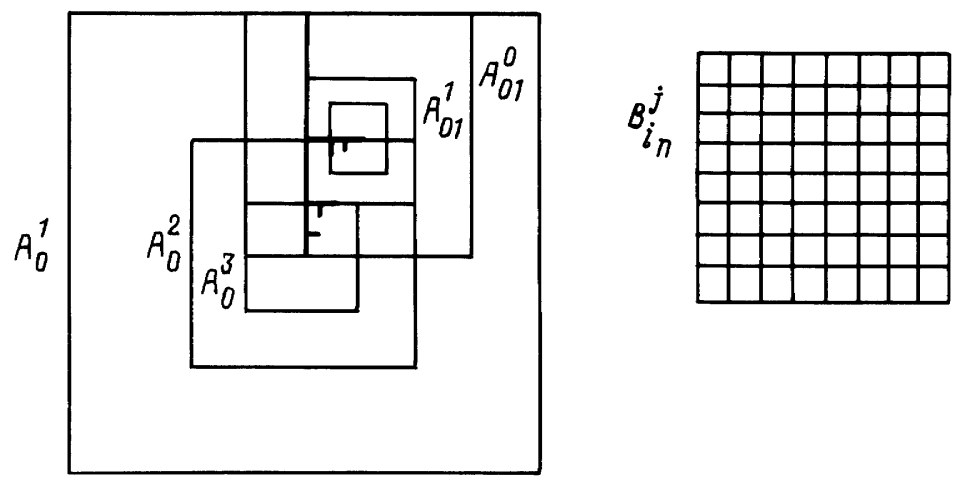

Рис. 2

Построим теперь более сложные чем $M$ и $N$ компактификации $Y$ и $Z$ множеств $L$ и $L \cup B$ соответственно с общим наростом. По определению $Y=f^{-1} M, Z=f^{-1} N$, где $f: Z \rightarrow N$ есть такое отображение, что $\left.f\right|_{L \cup B}=\mathrm{id}$, а для каждого $i f^{-1} q_{i}=I_{i}$, где $I_{i}=[0,1]_{i}$ - единичньй прямолинейньй отрезок; $L$ и $B$ мы определим как открытые подмножества пространства $Z$. Для того чтобы ввести топологию на множестве $Z$, осталось только определить базы окрестностей точек нароста $\bigcup_{i} I_{i}$. Топологическое пространство $Y$ определяется как подпространство $Z$.

Для каждого $i$ мы выберем специальную гомеоморфную копию $\eta_{i}\left(\mathbb{R}^{2} \backslash\left\{q_{i}\right\}\right)$ пространства $\mathbb{R}^{2} \backslash\left\{q_{i}\right\}$ и с ее помощью построим базу окрестностей точек элемента $I_{i}$.

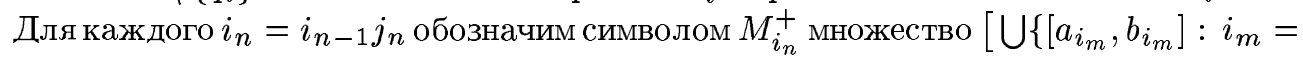
$\left.\left.i_{n} j_{n+1} \ldots j_{m}, n \leqslant m<\omega\right\}\right]_{\mathbb{R}^{2}}$, а символом $M_{i_{n}}^{-}$для $n \geqslant 1$ множество $\left[\left[a_{i_{n}}, b_{i_{n-1}}\right] \cup\right.$ $\left.\bigcup\left\{M_{i_{m}}^{+}: i_{m}=i_{n-1} j_{m}, j_{n}<j_{m}<\omega\right\}\right]_{\mathbb{R}^{2}}$. Эти множества являются "поддеревьями" “дерева" $M$ с началом в точке $a_{i_{n}}$. Поскольку при дальнейшем рассмотрении множества $M_{i_{n}}^{+}$и $M_{i_{n}}^{-}$не будут принципиально отличаться друг от друга, мы будем обозначать их обшим символом $M_{i_{n}}$. Каждому $M_{i_{n}}$ поставим в соответствие содержаший его замкнутый прямоугольник $T_{i_{n}}$ со сторонами, параллельными отрезкам из $M$, удовлетворяюший условию: если $M_{i_{n}}=M_{i_{n}}^{+}, i_{n}=i_{n-1} j_{n}$, то $\left(T_{i_{n}} \cap M\right) \backslash M_{i_{n}} \subset$ $\left(a_{i_{n-1}\left(j_{n}-1\right)}, a_{i_{n-1}\left(j_{n}+1\right)}\right)$; если $M_{i_{n}}=M_{i_{n}}^{-}$, то $\left(T_{i_{n}} \cap M\right) \backslash M_{i_{n}} \subset\left[a_{i_{n}}, a_{i_{n} 1}\right)$. Для каждого $T_{i_{n}}$ определим гомеоморфизм $\varphi_{i_{n}}: T_{i_{n}} \rightarrow T_{i_{n}}$, такой, что $\left.\varphi_{i_{n}}\right|_{\partial T_{i_{n}}}=\mathrm{id}$, $\operatorname{diam}\left(\varphi_{i_{n}} M_{i_{n}}\right) \leqslant \alpha_{i_{n}}$, где $\alpha_{i_{n}}$ - константа, которую мы выберем позднее. Помимо этого, функция

$$
\frac{\rho\left(\varphi_{i_{n}} x, \varphi_{i_{n}} y\right)}{\rho(x, y)}, \quad x \neq y, \quad x, y \in T_{i_{n}}
$$

ограничена. Описание конкретной конструкции такого отображения мы рассматривать не будем.

Пусть для данного $i P_{i}=p_{0}^{i} p_{1}^{i} p_{2}^{i} \ldots$ - однозначно определенная вьше ломаная, соответствуюшая точке $q_{i} \subset Q$ (рис. 3). От каждой вершины $p_{j}^{i}$ этой ломаной ответвляется 
один элемент $M_{i_{n}}$, который мы обозначим $M_{j}^{i}$. Учитьвая дизъюнктность семейства $\mathcal{T}_{i}=\left\{T_{i_{n}}: M_{i_{n}}=M_{j}^{i}, j<\omega\right\}$, определим гомеоморфизм $\varphi_{i}: \mathbb{R}^{2} \backslash\left\{q_{i}\right\} \rightarrow \mathbb{R}^{2} \backslash\left\{q_{i}\right\}$ через его сужения: $\left.\varphi_{i}\right|_{\mathbb{R}^{2} \backslash\left(\cup \mathcal{T}_{i} \cup\left\{q_{i}\right\}\right)}=\mathrm{id},\left.\varphi_{i}\right|_{T_{i_{n}}}=\varphi_{i_{n}}$ для каждого $T_{i_{n}} \in \mathcal{T}_{i}$. Очевидно, что $\varphi_{i} \mid P_{i}=\mathrm{id}$.
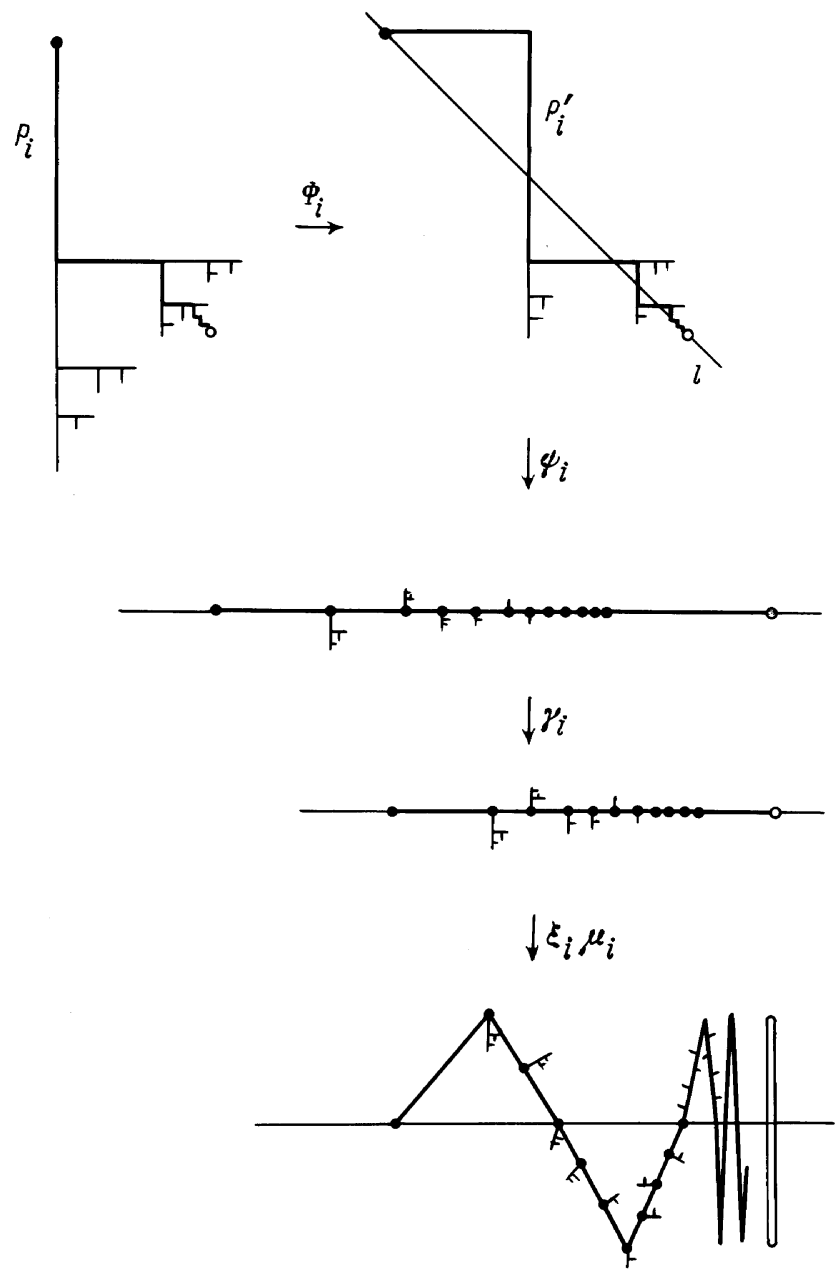

Рис. 3

Построим гомеоморфизм $\psi_{i}: \mathbb{R}^{2} \backslash\left\{q_{i}\right\} \rightarrow \mathbb{R}^{2} \backslash\left\{q_{i}\right\}$, “вьпрямляюший” ломаную $P_{i}$. Пусть $l$ - та из двух прямых в $\mathbb{R}^{2}$, проходящих через точку $q_{i}$ и составляющих угол $\pi / 4$ с $a_{0} b_{0}$, которая пересекает отрезок $a_{0} b_{0}$ ближе к точке $a_{0}$. Присоединим к ломаной $P_{i}$ дополнительное звено $p^{i} p_{0}^{i}$, перпендикулярное $p_{0}^{i} p_{1}^{i}$, где $p \in l$. Получим ломаную $P_{i}^{\prime}$. По определению $\left.\psi_{i}\right|_{P_{i}^{\prime}}$ есть ортогональное плоское проектирование $P_{i}^{\prime}$ на прямую $l$. Продолжим гомеоморфизм $\left.\psi_{i}\right|_{P_{i}^{\prime}}$ на $\mathbb{R}^{2} \backslash\left\{q_{i}\right\}$ как “сдвиг”. Через каждую точку $y \in \mathbb{R}^{2} \backslash\left\{q_{i}\right\}$ проведем прямую $l_{y}$, перпендикулярную $l$. Если $l_{y}$ пересекает $P_{i}^{\prime}$ в некоторой точке $z$, 
то $\psi_{i} y$ принадлежит прямой $z y, \rho\left(\psi_{i} y, \psi_{i} z\right)=\rho(y, z), \psi_{i} y$ лежит по ту же сторону от $\psi_{i} P_{i}^{\prime}$, что и $y$ от $P_{i}^{\prime}$. Если $l_{y} \cap P_{i}^{\prime}=\varnothing$, то $\psi_{i} y=y$.

Построим гомеоморфизм $\gamma_{i}: \mathbb{R}^{2} \backslash\left\{q_{i}\right\} \rightarrow \mathbb{R}^{2} \backslash\left\{q_{i}\right\}$. Выберем из последовательности $\left\{\psi_{i} p_{j}^{i}: j<\omega\right\}$ такую подпоследовательность $\left\{\psi_{i} p_{j_{k}}^{i}\right\}$, что $j_{k+1}-j_{k}=k$ для каждого $k<\omega$. Для всех $j, k, j_{k-1} \leqslant j<j_{k}$, сужения $\left.\gamma_{i}\right|_{\left[\psi_{i} p_{j}^{i}, \psi_{i} p_{j+1}^{i}\right]}$ определим как геометрическое подобие с такими коэффициентами, что $\rho\left(\gamma_{i} \psi_{i} p_{j}^{i}, \gamma_{i} \psi_{i} p_{j+1}^{i}\right)=\rho\left(\gamma_{i} \psi_{i} p_{\left(j_{k}-1\right)}^{i}\right.$, $\left.\gamma_{i} \psi_{i} p_{j_{k}}^{i}\right)=\rho\left(\psi_{i} p_{\left(j_{k}-1\right)}^{i}, \psi_{i} p_{j_{k}}^{i}\right)$. Кроме того, $\gamma_{i} \psi_{i} P_{i} \subset l,\left[\gamma_{i} \psi_{i} P_{i}\right]_{l}=\gamma_{i} \psi_{i} P_{i} \cup\left\{q_{i}\right\}$, отображение $\gamma_{i}$ сохраняет линейньй порядок узлов ломаной $P_{i}$. Разобьем $\mathbb{R}^{2}$ на полосы, разграниченные прямыми $l_{j}$, проходяшими через узлы $\psi_{i} p_{j}^{i}$ ломаной $\psi_{i} P_{i}$ и перпендикулярными ей. Сначала продолжим $\left.\gamma_{i}\right|_{\psi_{i} P_{i}}$ на каждую прямую $l_{j}$ как параллельный перенос. Пусть $\left[y_{1}, y_{2}\right]$ - какой-либо отрезок, параллельньй $l$ с концами, принадлежащими соседним разграничивающим прямым. Продолжим $\left.\gamma_{i}\right|_{\left\{y_{1}, y_{2}\right\}}$ до подобия $\left.\gamma_{i}\right|_{\left[y_{1}, y_{2}\right]}$. Сужение $\gamma_{i}$ на две крайние полосы, являюшиеся полуплоскостями, определим как изометрию. Гомеоморфизм $\gamma_{i}$ построен.

Определим гомеоморфизм $\mu_{i}$, действующий на $\mathbb{R}^{2} \backslash\left\{q_{i}\right\}$, оставляющий неподвижными все точки множества $\gamma_{i} \psi_{i} P_{i}$. Пусть $F$ есть объединение двух замкнутых в $\mathbb{R}^{2} \backslash\left\{q_{i}\right\}$ острых углов без общей вершины, находящейся в точке $q_{i}$, симметричных относительно $l$. Причем величины этих углов таковы, что семейство $\left\{A_{i_{n}}^{j}: a_{i_{n}}=p_{j}^{i}, j<\omega\right\}$ покрьвает $F$. Сужение $\mu_{i}$ на каждый из лучей, составляющих границу $F$ в $\mathbb{R}^{2} \backslash\left\{q_{i}\right\}$, есть параллельный перенос в направлении, перпендикулярном прямой $l$, причем множество

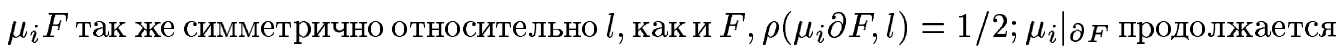
на $\mathbb{R}^{2} \backslash\left\{q_{i}\right\}$ подобно тому, как было продолжено отображение $\gamma_{i}$. На каждьй отрезок $\left[y_{1}, y_{2}\right],\left\{y_{1}, y_{2}\right\} \subset \partial F$, перпендикулярньй $l,\left.\mu_{i}\right|_{\left\{y_{1}, y_{2}\right\}}$ продолжается как подобие, на

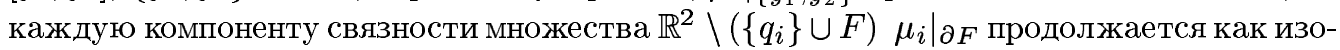
метрия. Заметим, что $\mu_{i}\left(R^{2} \backslash\left\{q_{i}\right\}\right)$ является плоскостью без единичного отрезка $I_{i}$.

Определим последний гомеоморфизм $\xi_{i}: \mathbb{R}^{2} \backslash I_{i} \rightarrow \mathbb{R}^{2} \backslash I_{i}$. Отображение, обратное к $\left.\xi_{i}\right|_{\gamma_{i} \psi_{i} P_{i}}$, представляет собой ортогональное проектирование на прямую $l$. Для каждого $k<\omega$ образ $\xi_{i}\left(\gamma_{i} \psi_{i}\left[p_{j_{k}}^{i}, p_{j_{k+1}}^{i}\right]\right)$ является отрезком, $\left.\xi_{i}\right|_{\gamma_{i} \psi_{i} p_{j_{2 k}}}=\mathrm{id}$, все точки $\xi_{i} \gamma_{i} \psi_{i} p_{j_{(4 k+1)}}$ лежат по одну сторону, а все точки $\xi_{i} \gamma_{i} \psi_{i} p_{j_{(4 k+3)}}$ лежат по другую сторону от прямой $l$ на расстоянии от $l$ равном $1 / 2$. Гомеоморфизм $\left.\xi_{i}\right|_{\gamma_{i} \psi_{i} P_{i}}$ продолжается на всю область определения аналогично тому, как это делалось в предыдущих двух случаях. На перпендикулярные прямой $l$ отрезки $\left[y_{1}, y_{2}\right], y_{1} \in \gamma_{i} \psi_{i} P_{i}, y_{2} \in \mu_{i} \partial F,\left.\xi_{i}\right|_{\left\{y_{1}, y_{2}\right\}}$ продолжается как подобие, $\left.\xi_{i}\right|_{R^{2} \backslash\left(I_{i} \cup \mu_{i} F\right)}=\mathrm{id}$.

Мы построили гомеоморфизм $\eta_{i}=\xi_{i} \mu_{i} \gamma_{i} \psi_{i} \varphi_{i}$. Произведя замыкание в $\mathbb{R}^{2}$, получим континуумы $\left[\eta_{i}\left(M \backslash\left\{q_{i}\right\}\right)\right]_{\mathbb{R}^{2}}=\eta_{i} M \cup I_{i}, I_{i}=[0,1]_{i},\left[\eta_{i}\left(N \backslash\left\{q_{i}\right\}\right)\right]_{\mathbb{R}^{2}}=\eta_{i} N \cup I_{i}$.

Определим базу окрестностей в $Z$ произвольной точки $x \in I_{i}$. Элементами базы будем считать множества вида $\left(U \cap I_{i}\right) \cup f^{-1} \eta_{i}^{-1}\left(U \cap \eta_{i}\left(N \backslash\left\{q_{i}\right\}\right)\right)$, где $U$ - какая-нибудь окрестность точки $x$ в $\mathbb{R}^{2}$. Топология в $Y$ и $Z$ введена. Нетрудно проверить, что $f$ - совершенное и монотонное непрерывное отображение. Поэтому $Z=f^{-1} N$ и $Y=f^{-1} M$ являются континуумами.

Подберем теперь постоянные $\alpha_{i_{n}}$ отображений $\varphi_{i_{n}}$ и мелкости сеток $B_{i_{n}}$ для всех $i_{n}=j_{0} j_{1} \ldots j_{n}$. Назовем коэффициентом искажения $\varepsilon_{h}(E)$ непрерьвного отображе- 
ния $h: \mathbb{R}^{2} \rightarrow \mathbb{R}^{2}$ на множестве $E \subseteq \mathbb{R}^{2}$ величину

$$
\sup _{\left\{y_{1}, y_{2}\right\} \subseteq E} \frac{\left|\rho\left(h\left(y_{2}\right), h\left(y_{1}\right)\right)-\rho\left(y_{2}, y_{1}\right)\right|}{\rho\left(y_{2}, y_{1}\right)}, \quad y_{1} \neq y_{2},
$$

которая может принимать бесконечное значение. Конструкция описанных вьше гомеоморфизмов такова, что если $h_{i}$ - одно из отображений $\varphi_{i}, \psi_{i}, \gamma_{i}, \mu_{i}, \xi_{i}$, то для каждого множества $E \subset \mathbb{R}^{2} \backslash Q$ такого, что $\rho(E, Q)>0$, выполняются соотношения $\varepsilon_{h_{i}}(E)<$ $\varepsilon(E)<\infty$ и $\rho\left(h_{i}(E), I_{i}\right)>\rho(E)>0$, где $\varepsilon(E)$ и $\rho(E)$ - не зависяшие от $i$ величины. Кроме того, каждое $E$ содержит конечное число вершин ломаных $P_{i}$. Конкретное доказательство этих свойств мы проводить не будем. Пользуясь этими фактами, подберем для каждого $i_{n}$ такую константу $\alpha_{i_{n}}$ отображения $\varphi_{i_{n}}$, чтобы для всех $i$ и $j$ таких, что $p_{j}^{i}=a_{i_{n}}$, вьполнялись свойства:

$$
\begin{aligned}
& \operatorname{diam}\left(\eta_{i} M_{j}^{i}\right)<\rho^{2}\left(\eta_{i} p_{j}^{i}, \eta_{i} p_{k}^{i}\right) \quad \text { для всех } k \neq j \\
& \operatorname{diam}\left(\eta_{i} M_{j}^{i}\right)<\rho^{2}\left(\eta_{i} p_{j}^{i},\left[\eta_{i} p_{k}^{i}, \eta_{i} p_{k+1}^{i}\right]\right) \quad \text { для всех } p_{k}^{i} \neq p_{j}^{i} \neq p_{k+1}^{i} .
\end{aligned}
$$

Ячейкой сетки $\eta_{i} B$ мы считаем образ какой-либо ячейки сетки $B$. Мелкость $\delta$ определяется аналогично тому, как это было сделано для сетки $B$. Так как для каждых $i_{n}$ и $j$ множество $D_{i_{n}}^{j}=A_{i_{n}}^{j} \backslash\left(\bigcup_{m>n, k, i_{m}} A_{i_{m}}^{k} \cup A_{i_{n}}^{j+1}\right)$ находится на положительном расстоянии от $Q$, пользуясь вьшеупомянутыми свойствами гомеоморфизмов $\eta_{i}$, для каждых заданных $i_{n}$ и $j$ мы выберем сетку $B_{i_{n}}^{j}$ настолько мелкой, чтобы для всех $i$ были верны следующие неравенства:

$\delta\left(\eta_{i} D_{i_{n}}^{j}\right)<\operatorname{diam}^{2}\left(\eta_{i} M_{k}^{i}\right)$ для всех таких $k$, что $M_{k}^{i} \cap D_{i_{n}}^{j} \neq \varnothing ;$

$\delta\left(\eta_{i} D_{i_{n}}^{j}\right)<\rho^{2}\left(\eta_{i} p_{m}^{i}, \eta_{i} p_{k}^{i}\right)$ для всех $k \neq m$ и таких $m$, что $\eta_{i} p_{m}^{i}$ - ближайшая вершина ломаной $\eta_{i} P^{i}$ к какой-либо точке из $\eta_{i} D_{i_{n}}^{j}$;

$\delta\left(\eta_{i} D_{i_{n}}^{j}\right)<\rho^{2}\left(\left[\eta_{i} p_{m}^{i}, \eta_{i} p_{m+1}^{i}\right],\left[\eta_{i} p_{k}^{i}, \eta_{i} p_{k+1}^{i}\right]\right)$ для всех таких $m$ и $k$, что $\left[\eta_{i} p_{m}^{i}, \eta_{i} p_{m+1}^{i}\right]$ - ближайшее звено ломаной $\eta_{i} P_{i}$ к какой-либо точке из $\eta_{i} D_{i_{n}}^{j}, \mathrm{a}\left[p_{k}^{i}, p_{k+1}^{i}\right] \cap\left[p_{m}^{i}, p_{m+1}^{i}\right]=$ $\varnothing$. Поскольку $\cup \mathcal{A} \backslash Q=\bigcup_{i_{n}, j} D_{i_{n}}^{j}$, мы добились того, что для любой точки $x \in \cup \mathcal{A} \backslash Q$ и каждого $i$ вьполняются следующие свойства:

$$
\delta\left(\eta_{i} x\right)<\operatorname{diam}^{2}\left(\eta_{i} M_{j}^{i}\right)
$$

для всех $j$ таких, что $M_{j}^{i} \ni x$;

$$
\delta\left(\eta_{i} x\right)<\rho^{2}\left(\left[\eta_{i} p_{m}^{i}, \eta_{i} p_{m+1}^{i}\right],\left[\eta_{i} p_{k}^{i}, \eta_{i} p_{k+1}^{i}\right]\right)
$$

где $\left[\eta_{i} p_{m}^{i}, \eta_{i} p_{m+1}^{i}\right]$ - ближайшее к $x$ звено ломаной $\eta_{i} P_{i}$, для всех таких $k$, что $\left[p_{k}^{i}, p_{k+1}^{i}\right] \cap$ $\left[p_{m}^{i}, p_{m+1}^{i}\right]=\varnothing$.

Множества $Y$ и $Z$ построены. Пусть $g: Y \rightarrow g Y$ - какой-нибудь гомеоморфизм, обладающий свойством: $\left.g\right|_{\bigcup_{i} I_{i}}=\mathrm{id}, g L \cap(L \cup B)=\varnothing$. Окончательную конструкцию - пространство $X$ определим как множество $Z \cup g Y$ с топологией прямой суммы топологических пространств $Z$ и $g Y$. Так как $Z$ и $Y$ - континуумы и $Z \cap g Y \neq \varnothing, X$ тоже является континуумом. 
Доказательство. Докажем, что пространство $X$ локально связно и периферически счетно. Поскольку $X=L \cup B \cup g L \cup \bigcup_{i} I_{i}$, а локально связное и периферически конечное подмножество $L \cup B \cup g L$ открыто в $X$, достаточно доказать эти свойства для точек множества $\bigcup_{i} I_{i}$.

Свойства (1)-(4), а также тот факт, что $\rho\left(\eta_{i} p_{k}^{i}, \eta_{i} p_{k+1}^{i}\right) \rightarrow 0$ при $\rho\left(\eta_{i} p_{k}^{i}, I_{i}\right) \rightarrow 0$ или $k \rightarrow \infty$, позволяют построить произвольно малую окрестность $V$ точки $\eta_{i} x=x$ в $\eta_{i}\left(N \backslash\left\{q_{i}\right\}\right) \cup I_{i}$, связную и со счетной границей. Детальное доказательство возможности такого построения мы здесь рассматривать не будем. Окрестность $V$ строится следуюшим образом.

Пусть $H$ - открытьй в $\mathbb{R}^{2}=\eta_{i}\left(\mathbb{R}^{2} \backslash\left\{q_{i}\right\}\right) \cup I_{i}$ прямоугольник, содержащий точку $x$, симметричный относительно прямой, содержащей $I_{i}$. Выберем горизонтальный и вертикальньй размеры прямоугольника $H$ не превосходящими заданной произвольно малой величины, достаточно малыми и соотносящимися таким образом, чтобы результаты дальнейших построений обладали соответствующими свойствами.

Каждая компонента связности множества $\eta_{i} P_{i} \cap H$ в силу выбора $H$ содержит хотя бы одну вершину $\eta_{i} p_{j}^{i}$. Для каждого такого $j$, что $\rho\left(\eta_{i} p_{j}^{i}, H\right)<2 \operatorname{diam}\left(\eta_{i} M_{j}^{i}\right)$, определим открытый прямоугольник $S_{j}^{i} \supset \eta_{i} M_{j}^{i}$, пересекающий $H$, со сторонами, параллельными сторонам $H$, для которого $\operatorname{diam}\left(S_{j}^{i}\right)<3 \operatorname{diam}\left(\eta_{i} M_{j}^{i}\right)$. Присоединим к $H$ все такие квадраты. Получим открытое множество $G$, которое представляет собой сцепленную систему связных множеств и потому связно. Прямоугольники $S$ мы подберем таким образом, чтобы каждая компонента связности множества $\eta_{i} P_{i} \cap G$ содержала компоненту связности множества $\eta_{i} P_{i} \cap H$. Кроме того, $\rho\left(\eta_{i} M_{j}^{i}, G\right)>\operatorname{diam}\left(\eta_{i} M_{j}^{i}\right)$ для каждого $\eta_{i} M_{j}^{i} \nsubseteq G$. Все это достигается за счет малости размеров $H$ и за счет быстрого уменьшения $\operatorname{diam}\left(\eta_{i} M_{j}^{i}\right)$ при $\rho\left(\eta_{i} p_{j}^{i}, I_{i}\right) \rightarrow 0$.

Ячейкой сетки $\eta_{i} B$ мы будем называть образ какой-либо ячейки сетки $B$ под действием гомеоморфизма $\eta_{i}$. Мелкость для $\eta_{i} B$ определяется точно так же, как это было сделано для $B$. Для каждой ячейки $C$ сетки $\eta_{i} B$ определим открытый многоугольник $S(C) \supset C$, удовлетворяюший условиям: множество $S(C) \cap \eta_{i}\left(B \cup P_{i}\right)$ связно, множество $\partial S(C) \cap \eta_{i}\left(N \backslash\left\{q_{i}\right\}\right)$ конечно, $\partial S(C) \cap \eta_{i}\left(Q \backslash\left\{q_{i}\right\}\right)=\varnothing, \operatorname{diam}(S(C))<2 \operatorname{diam}(C)$. Обозначим через $G_{1}$ и $G_{2}$ пересечения $G$ соответственно с левой и правой открытыми полуплоскостями, на которые разбивает плоскость прямая, содержашая отрезок $I_{i}$. Мы имеем $G=G_{1} \cup\left(H \cap I_{i}\right) \cup G_{2}$. Построим счетные покрытия $\mathcal{S}_{1}=\left\{S(C): C \in \mathcal{C}_{1}\right\}$ и $\mathcal{S}_{2}=\left\{S(C): C \in \mathcal{C}_{2}\right\}$ множеств $G_{1}$ и $G_{2}$ соответственно. Покрытия $\mathcal{S}_{1}$ и $\mathcal{S}_{2}$ обладают следуюшими свойствами. Для каждой ячейки $C \in \mathcal{C}_{1} \cup \mathcal{C}_{2} \operatorname{diam}(C) \leqslant \rho^{2}\left(y, I_{i}\right)$ для некоторой точки $y \in C \cap G \neq \varnothing$. Множества $\cup \mathcal{S}_{k} \cap \eta_{i}\left(B \cup P_{i}\right), k=1,2$, связны. Каждая компонента связности множества $\cup \mathcal{S}_{1} \cap \eta_{i} P_{i}$ содержит компоненту связности множества $G \cap \eta_{i} P_{i}$. Каждое $\eta_{i} M_{j}^{i}$ либо является подмножеством множества $G$, либо не пересекает $\cup \mathcal{S}_{1}$. Эти свойства достигаются выбором прямоугольника $H$ за счет быстрого уменьшения $\delta(y)$ при $\rho\left(y, I_{i}\right) \rightarrow 0$.

Докажем, что окрестность $V=\left(\cup \mathcal{S}_{1} \cup \cup \mathcal{S}_{2} \cup H\right) \cap\left(\eta_{i}\left(N \backslash\left\{q_{i}\right\}\right) \cup I_{i}\right)$ связна. Как уже известно, множество $V_{2}=V \cap\left(\cup \mathcal{S}_{2}\right)=\left(\cup \mathcal{S}_{2}\right) \cap \eta_{i}\left(B \cup P_{i}\right)$ связно; $V_{1}=V \cap$ $\left(\cup \mathcal{S}_{1}\right)=\left(\cup \mathcal{S}_{1}\right) \cap\left(\eta_{i}\left(B \cup P_{i}\right) \cup \cup\left\{M_{j}^{i}: j<\omega\right\}\right)$, но каждое $M_{j}^{i}$ либо не пересекает $V_{1}$, либо является подмножеством $V$ и пересекает $\eta_{i}\left(B \cup P_{i}\right)$. Следовательно, $V_{1}$ связно. Поскольку $\left[V_{1}\right]_{R^{2}} \cap\left[V_{2}\right]_{R^{2}} \cap H \cap I_{i} \neq \varnothing, V$ тоже связно.

Докажем, что множество $\partial V$ счетно. Поскольку семейства $\mathcal{S}_{1}$ и $\mathcal{S}_{2}$ счетны, а каждое 
множество $\partial S(C)$ конечно, множество $\partial V \backslash I_{i}$ счетно. Из свойств $\mathcal{S}_{1}$ и $\mathcal{S}_{2}$ следует, что $\sup _{y \in A\left(\rho_{0}\right)} \rho(y, H) \rightarrow 0$ при $\rho_{0} \rightarrow 0$, где $A\left(\rho_{0}\right)=\left\{y \in V \backslash H: \rho\left(y, I_{i}\right)<\rho_{0}\right\}$. Поэтому, очевидно, что $\partial V \cap I_{i}=\partial H \cap I_{i}$ состоит из двух точек. Счетность $\partial V$ доказана.

Вполне очевидно, что открытое множество $f^{-1} \eta_{i}^{-1} V=W$, так же как и $V$, связно и имеет счетную границу в $Z$, а также то, что окрестность $U=W \cup g(W \cap Y)$ имеет счетную границу в $X$. Каждая компонента связности множества $V \cap \eta_{i}\left(M \backslash\left\{q_{i}\right\}\right)$ содержит хотя бы одну точку множества $\eta_{i} Q$. Следовательно, каждая компонента связности множества $W \cap Y=f^{-1} \eta_{i}^{-1} V \cap f^{-1} M$ и соответственно множества $g(W \cap Y)$ содержит хотя бы один элемент $I_{i}$, принадлежащий связному множеству $W$. Поэтому окрестность $U$ связна.

Такие множества как $U$ составляют базу окрестностей точки $x$ в пространстве $X$. Периферическая счетность и локальная связность $X$ доказаны.

Докажем, что пространство $X$ не является линейно связным. Пусть $l$ - линия в $X$, соединяюшая точку $x \in g Y \backslash \bigcup_{i} I_{i}$ с точкой $y \in Z$. Поскольку подмножества $Z \backslash \bigcup_{i} I_{i}$ и $g Y \backslash \bigcup_{i} I_{i}$ открыты, $X=\bigcup_{i} I_{i} \cup Z \backslash \bigcup_{i} I_{i} \cup g Y \backslash \bigcup_{i} I_{i}$ и $Z \cap g Y \backslash \bigcup_{i} I_{i}=\varnothing$, то $l \cap\left(\bigcup_{i} I_{i}\right) \neq \varnothing$, так как $X$ связно. Пусть $z \in I_{j}$ - "ближайшая по линии $l$ " к точке $x$ точка множества $l \cap\left(\bigcup_{i} I_{i}\right)$. Отсюда следует, что $g Y$, а следовательно, и $Y$ содержит линию $l^{\prime}, l^{\prime} \cap\left(\bigcup_{i} I_{i}\right)=\{z\}$, соединяющую точку $x \notin I_{j}$ с точкой $z \in I_{j}$, что очевидно невозможно.

\section{СПИСОК ЦИТИРОВАННОЙ ЛИТЕРАТУРЫ}

[1] Nikiel J., Tuncali H. M., Tymchatyn E. D. A locally connected rim-countable continuum which is the continuous image of no arc // Top. Appl. 1991. V. 42. P. 83-93.

Московский государственный университет им. М.В. Ломоносова

Поступило

25.12 .1995

Исправленный вариант

01.10 .1998 